Journal of Computer Science 7 (6): 909-916, 2011

ISSN 1549-3636

(C) 2011 Science Publications

\title{
Design and Development of Robot Hand System
}

\author{
${ }^{1}$ Panchumarthy Seetharamaiah, ${ }^{2}$ Mandapati Venkateswara Rao and \\ ${ }^{3}$ Geddapu Venkata Satyanarayana \\ ${ }^{1}$ Department of Computer Science and Systems \\ Engineering, Andhra University, \\ ${ }^{2}$ Department of Information Technology, GITAM University, \\ ${ }^{3}$ Department of Information Technology, \\ Raghu Institute of Technology, Dakamarii, Visakhapatnam, India
}

\begin{abstract}
Research on robot hand design is being carried out to accommodate a variety of tasks such as grasping and manipulation of objects in the field of industrial applications, service robots and rehabilitation robots. Problem statement: To design and develop a microcontroller-based four fingered robotic hand with a simple and minimal control strategy to pick and place application with object detection by simple IR sensor logic. Approach: The methodology is based on anthropomorphic design with three fingers and an opposing thumb. Each finger has three links and three double revolute joints. Each finger is actuated by a single opposing pair of tendons. The robot hand system is interfaced to microcontroller with software control by means of 14 independent commands for the motion of joints: close and open for fore finger, middle finger, ring finger and thumb finger and wrist up and down, base clockwise and counter clockwise, pick and place and home position. The tendoning system and wireless feedback logic provide the hand with the ability to confirm to object topology and therefore providing the advantage of using a simple control structure. Results: Reliable grasping and releasing is achieved with simple control mechanisms and IR sensors/push-button switches. The hand can pick a variety of objects with different surface characteristics and shapes without having to reconstruct its surface description. Picking of the object is successfully completed as long as the object is within the workspace of the hand and placed the object at the desired position within the workspace by relevant software control using keyboard commands. Conclusion: Hardware and software development of microcontroller-based four-fingered robotic hand is addressed. Details of hand control software for mainly pick and place applications are presented. Results of the experimental work for pick and place application of different objects is enumerated.
\end{abstract}

Key words: Hand control software, object hunting, IR sensors, Four Fingered Robotic Hand (FFRH), In-System Programming (ISP), cylindrical robot, microcontroller-based, opposing thumb, counter clockwise, Degrees Of Freedom (DOF)

\section{INTRODUCTION}

A robot is a reprogrammable multifunctional manipulator designed to move material, parts, tools or specialized devices through variable programmed motions for the performance of a variety of tasks. Robots are in need in industrial field where tasks and operation are done with high speed and accuracy and in non-industrial fields where assistance to personal and increased convenience are needed. The needs for robots have recently been changed from factory automation to human friendly robot system. With increasingly aging societies, the realization of robot hand that assists human activities in daily environments such as in offices, homes and hospitals are required. In the future, a humanoid robot, which can walk biped ally and perform skilful tasks by dual-arm with hands, would be one of ultimate robots that have an ability of cooperative and coexistence with humans, because of anthropomorphism, friendly design, applicability of locomotion, behavior within the human living environments and so on. Over the past few years, a number of robotic hands have been developed (Cho et al., 2006; Odeh et al., 2009; Mayuko et al., 2010) for dexterous and skilful grasping applications in medical, welfare, space, industrial and virtual environments. Robot hands (Xu et al., 2009; Wu et al., 2010; Liu et al., 2008) should be compact and light weight. They

Corresponding Author: Panchumarthy Seetharamaiah, Department of Computer Science and Systems Engineering, Andhra University, Visakhapatnam, India 
should also be able to move their fingers quickly before grasping an object and be able to exert a large grasp force when grasping the object. To meet these requirements with a fixed reduction ratio, the hand needs to use large motors, which makes the hand large and heavy. If the performances of electromagnetic motors are improved, the speed and force performances could be improved without increasing the weight of the hand.

The goal of advanced robotics is to develop combined computer and mechanical structure which can perform operations in a manner analogous to human beings. A mechanical four or five fingered replica of the human hand is the best choice for such a robot because the world is designed around five finger hands. A robot with such an end-effector would be capable of very robust operations.

Robots can be classified according to their method of control pathway, structural design, or level of technology. The two major types of control are servo and non-servo. The path way of the robot may be either point- to-point or continuous. The volume of the workspace of a robot is rectangular, cylindrical, spherical, or jointed spherical. Finally, a robot may be classified based on its number of axes and its level of sophistication in respect of end effectors/ grippers. The gripper is similar to the human hand just as the hand grasps the tool to perform the work. The shape of the gripper is determined by the task it has to perform. Twopronged or general grippers are used to pick up cylindrical and cubical objects, three-pronged grippers are used on spherical objects and specialized grippers can be designed for unique tasks. Object grasping techniques which do not need the computation of three-dimensional surface information are essential for robotics applications where computational power may be limited, for example on mobile delivery and retrieval robots.

The aim of this paper is to design and implement a Four Fingered Robotic Hand (FFRH) for providing a simple reflexive grasp that can be utilized for a wide variety of objects. The FFRH is designed based on servo, point-to-point and cylindrical robot structure with fourpronged grippers (four fingers). This approach is focusing primarily on the task of grasping objects and not that of manipulating or assembling objects. This type of a grasping device has a variety of applications in object retrieval systems for the handicapped, planetary, underwater exploration and robotic surgery. To fulfill the objective, authors designed a new general purpose FFRH that grasps a variety of objects with a simple control scheme that has 14 independent commands for fingers, wrist and base including home position and pick and place. FFRH is designed to have size and force generation capabilities similar to a human and can be expected to manipulate variety of objects. Nevertheless, FFRH is not to manipulate objects, but only to grasp objects reliably. Therefore, there is no need of all the complex characteristics of the human hand or many existing anthropomorphic robot hands. The methodology adopted is a simplified anthropomorphic design with three fingers and an opposing thumb with double revolute joints, almost similar in structure to the human hand. Each finger is actuated by a single opposing pair of tendons. One tendon curls the finger towards the palm and the other opposing tendon extends the finger away from the palm so as to grasp the objects. In this paper, the design and implementation details of microcontroller-based fourfingered robotic hand are presented. The hardware and software implementations with mechanical structure of the hand are explained. The control algorithm based on microcontroller system with drivers for FFRH and wireless feedback logic for activation and deactivation is presented. Finally, the results of the experimentation and ideas for future work are outlined.

\section{MATERIALS AND METHODS}

The FFRH has three main parts, Robot hand structure, Robot hand controller hardware and Robot hand controller software.

Robot hand structure: The design of mechanical hand structure for FFRH incorporates four digits/fingers: three fingers and one thumb, as shown in Fig. 1 Three digits are positioned at the corners of an inverted triangle and one digit is at the center of base of the triangle, since this geometry leads naturally to stable finger contact positions for an enclosing grasp. Each finger consists of three rigid links (the proximal, intermediate and distal phalanges) constructed from two parallel plates. The phalanges are connected by three joints (the proximal, intermediate and distal joints) which have parallel axes of rotation and are responsible for curling the finger tip toward the palm. The dimensions of FFRH are selected to be approximately the size of an adult human hand. The phalange lengths, $\mathrm{P}_{1}, \mathrm{P}_{2}$ and $\mathrm{P}_{3}$, phalange width, $\mathrm{w}$ and the distance between the finger and the thumb, $\mathrm{d}_{\mathrm{f}}$, were selected as shown in Table 1 . The distance between the two fingers, $d_{f f}$, was computed to be the distance between the fourth and index finger to give the largest human finger displacement. The thickness of the supports t, was chosen for availability and strength. To reduce construction costs, we configured the three fingers identically and the thumb to have identical link lengths as the proximal and intermediate links of the fingers. 
Table 1: Robotic hand parameters

\begin{tabular}{lc}
\hline Distance between joints & Value $(\mathrm{mm})$ \\
\hline Palm to proximal joint- $\mathrm{P}_{0}$ & 19 \\
Proximal to intermediate joint- $\mathrm{P}_{1}$ & 47 \\
Intermediate to distal joint- $\mathrm{P}_{2}$ & 25 \\
Distal joint to digit tip- $\mathrm{P}_{3}$ & 19 \\
Joint to end of phalange-l & 9 \\
Phalange width-w & 19 \\
Finger to Finger- $\mathrm{d}_{\mathrm{ff}}$ & 44 \\
Thumb and fingers- $\mathrm{d}_{\mathrm{ft}}$ & 75 \\
Support thickness- $\mathrm{t}$ & 3 \\
Proximal joint pulley radius- $\mathrm{R}_{1}$ & 9 \\
Intermediate joint pulley radius- $\mathrm{R}_{2}$ & 8 \\
Distal joint pulley radius- $\mathrm{R}_{3}$ & 6 \\
\hline
\end{tabular}
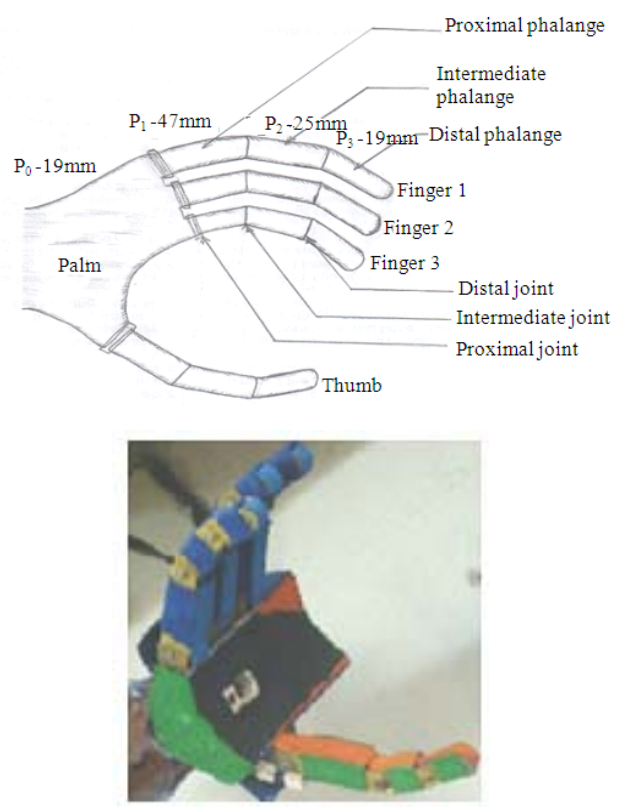

Fig. 1: Four fingered robot hand-FFRH
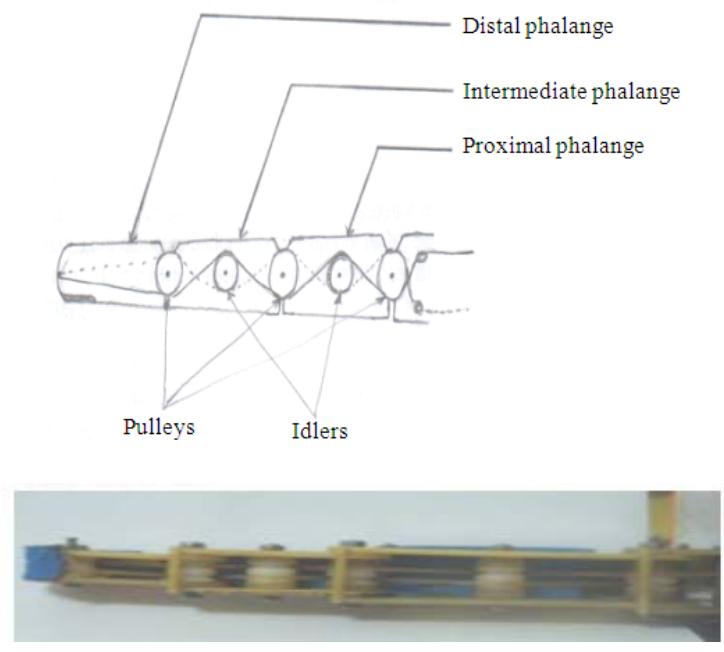

Fig. 2: Finger structure
Each digit is controlled by a single antagonistic pair of tendons which are routed over a system of pulleys and idlers as shown in the Fig. 2.

The pulleys act as routers to create angular displacement of the link, but do not transfer any torque to the joint. Each phalange consists of a pulley located at the joint and an idler located at the center of the phalange. The opposing tendon controlling the finger is routed in opposite direction over the pulley and idler. The path of the tendon to this system works as a differential mechanism. One end of each tendon is attached to the tip of the distal phalange and the other is wound about a spool attached to the shaft of a single remotely located geared DC motor. Each finger is controlled by one reversible geared DC motor. Using IR sensors, the finger joint motion limits are monitored by microcontroller ports and also the finger can be locked at any stage within the workspace. The tendons in each finger are wound in both directions depending on the DC motor direction. The IR sensors provide the feedback information from the hardware back to the control software. This is necessary to perform a closed loop control of the fingers or a grasped object.

Material selected for finger plates is epoxy since it is strong, rigid, lightweight, relatively in expensive and easy to machine. We also choose epoxy for the arm, wrist and palm plates since it is not as susceptible to wear. Dial cords with small diameter plastic tubes as sleeves are used for the tendons of the digit since these are flexible.

Object hunting methodology: Many researchers have outlined algorithms for grasping objects of various shapes with finger grippers (Ciocarlie and Allen, 2009; Grecu et al., 2009). While this is possible, the robot must compute where to position the jaws relative to the surface and center of mass of the object. To hunt for the presence of an object on workspace, IR sensor is placed at the central position of the palm as shown in the Fig. 3. In a conceptual representation.

Upon power-on condition, the robot hand moves to the home position and by issuing a Pick and Place (P\&P) command from the keyboard, the hand starts rotating in 360 degrees via base motor control. Once the IR sensor in the palm of hand detects obstruction due to the presence of an object in the study table, it identifies the first edge of the object and continues to move for detection of second edge. Upon detecting the second edge, it moves back to the mid position and starts grasping the object. After completely grasping the object using four fingers, the hand moves to the predefined destination on the work table and releases the object. 


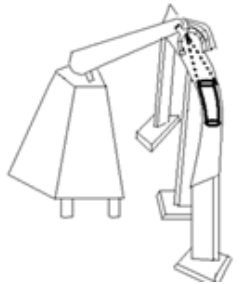

(a)

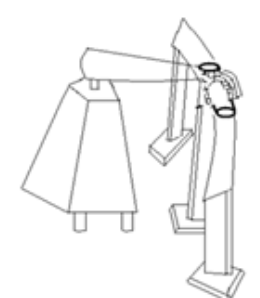

(b)

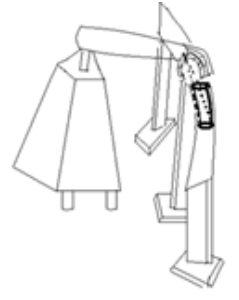

(c)
Fig. 3: Object hunting process (a) 1st edge detection (b) 2nd edge detection (c) Back to Mid Position (Ready to Pick)

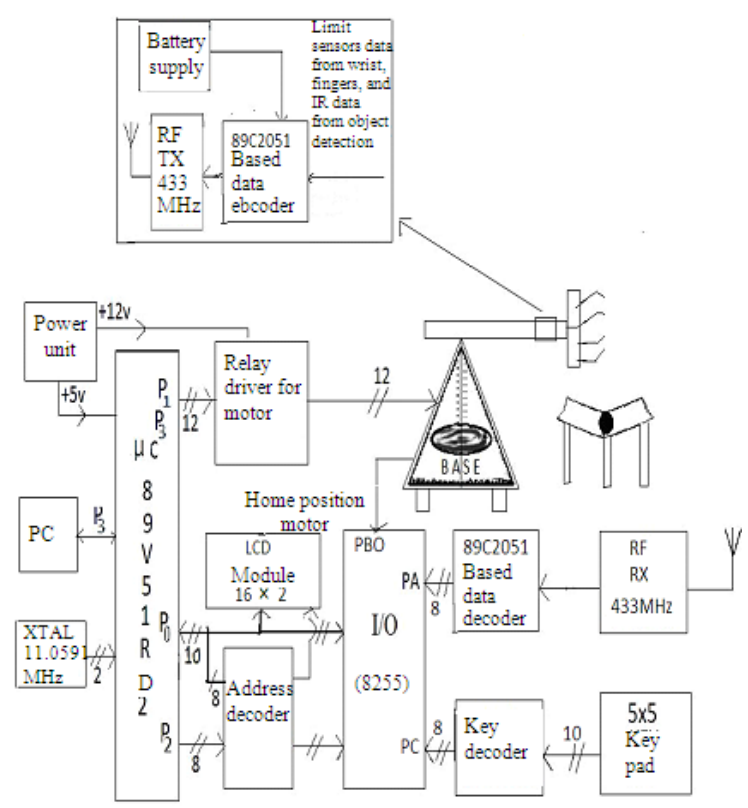

(a)

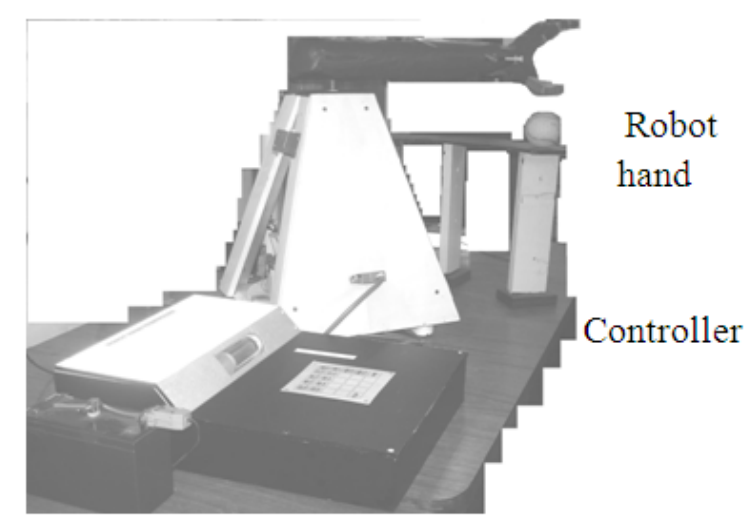

(b)

Fig. 4:(a) Block Diagram of Complete Four Fingered Robot Hand System (b) Practical Four Fingered Robot Hand with Controller
All these tasks are implemented by single command called P\&P key on the keyboard interfaced to the hand system.

FFRH Controller Hardware: The FFRH system has three main parts namely Robot Hand, Controller and Sensor Feedback unit. The Robot Hand consists of three fingers and an opposing thumb and are connected internally to the geared DC motors, for their movement. The FFRH system, whose block diagram is shown in Fig. 4, consists of a microcontroller P89V51RD2BN operating at $11 \mathrm{MHz}$, relay-based geared dc-motor drivers, an RS232 port to communicate with a PC, an LCD display for debugging, an In-System Programming (ISP) port for downloading programs from a PC to the 64-KB flash memory, 8 data lines from limit sensors of hand via 89C2051 microcontroller-based encoder/decoder and RF transmitter/receiver to control the limits of movement of hand, 5×5 key matrix interface using 8255 port PC for hand's control commands.

All the joints of the hand including the base are controlled by geared DC motors with $10 \mathrm{rpm}$ and $1 \mathrm{Kg}-$ $\mathrm{cm}$ torque using strong gut type wires wound from the shaft to the finger joint pulleys and idlers and relay drivers interfaced to full P1 port bits and half of the P3 port bits of P89V51RD2BN. A Programmable Peripheral Interface-PPI 8255 is interfaced to P89V51RD2BN at memory mapped i/o addresses fe00h to fe03h for ports PA,PB,PC and CP. Port PC is configured as input from $5 \times 5$ key matrix, port bit $\mathrm{PB} 0$ is configured as input bit from home position sensor(IR $\mathrm{TX} / \mathrm{RX}$ ), port PA is configured as input from port P1 of 89C2051 that has received sensor data(Limit sensors-4 push-button switches for 4 fingers, 3 IR TX/RX for wrist positions-upper/lower/mid) from $433 \mathrm{MHz}$ RF FSK receiver. The $433 \mathrm{MHz}$ RF FSK receiver is continuously receiving sensor data from $433 \mathrm{MHz} \mathrm{RF}$ FSK transmitter and/89C2051 port P1 that in turn continuously getting data from sensors placed in hand. Base motor is used to move hand in 360 degrees in either direction programmed by key commands (basecw, base-ccw). Four fingers use four geared DC motors/drivers with relays, for motion control of finger joints with pitch of 45 degrees maximum. Wrist geared DC motor drives the wrist with roll and pitch of maximum 160 degrees. The input from finger limit sensors placed on palm is used to control the free rotation of the finger. IR sensor placed at the center of the Palm is used to sense the presence of an object. The operation of microcontroller-based robot hand is achieved through control program code burned in flash memory of microcontroller. 


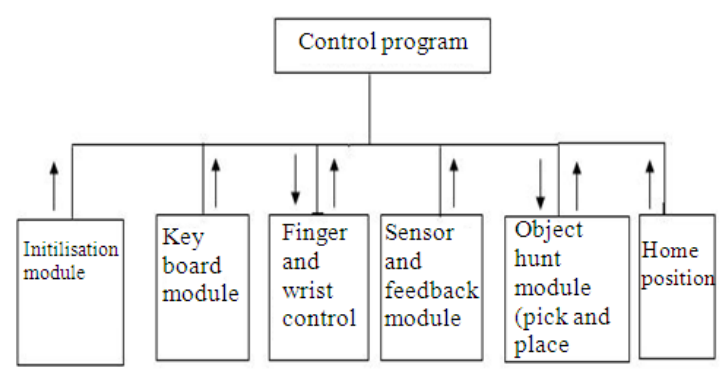

Fig. 5: FFRH controller software structure

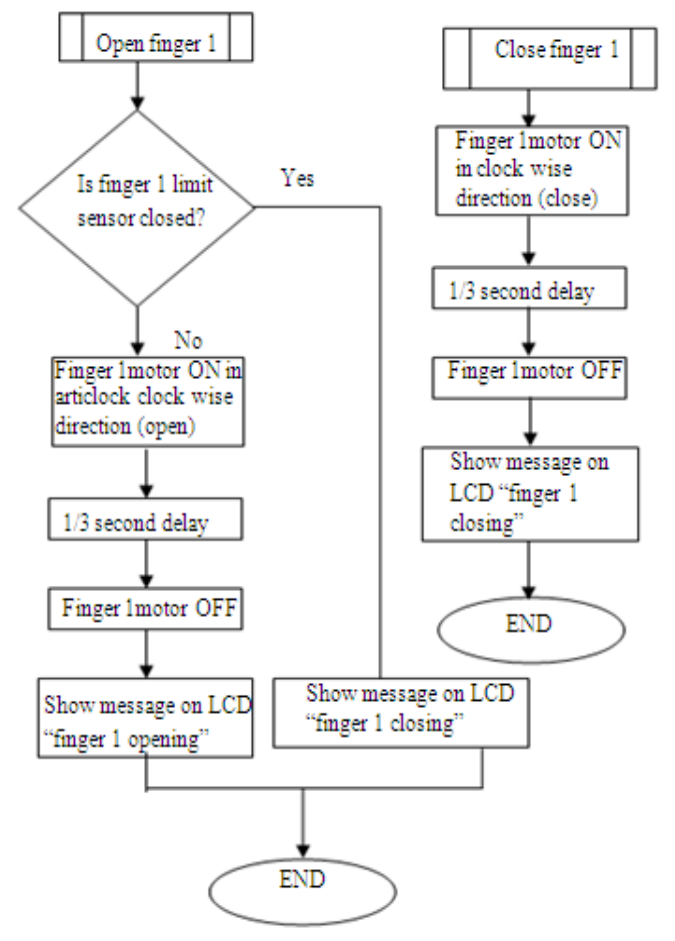

Fig. 6: Flowchart for Finger 1 operation

FFRH controller software: In terms of flexibility, a modular software structure is essential for a research system like the hand system presented here. Software is based on the task level programming environment for real time control with the keyboard commands. The FFRH is controlled by 14 independent commands embedded in the hand controller memory (Flash memory of 89V51 $\mu \mathrm{C}$ ) activated by keyboard, for the following joints: Fore finger close and open, Middle finger close and open, Ring finger close and open, Thumb finger close and open, Wrist up and down, Base clockwise and counter clockwise, Pick and Place and Home position. Figure 5 shows the structure chart of control program of the FFRH.
The control software for FFRH is developed in 8051 ASM language. Software tool used to debug the program is Keil-IDE. Hex Code generated by the KeilIDE is burned into 89V51 using the Flash Magic Software. Six major software modules such as initialization, keyboard, fingers/wrist control, sensor feedback, object hunting followed by pick and place and home position are incorporated in the hand control program. The $5 \times 5$ key matrix polled real-time firmware flowchart is shown in Fig. 6. For one finger operation and operation of other fingers is exactly similar.

The control program upon power-on waits for task initiated by the key command and executes the command and waits for the next command. Home position key command from keyboard drives the hand to a predefined position using position sensor by IR sensor logic. Pick and Place command key drives the hand from its current position in clockwise continuously 360 degrees and hunts for an object using IR sensor placed in the center position of the palm. Once object's first edge is detected, it hunts for last edge. Then it moves back to the center position of the object and starts picking the object with finger limiting sensors and moves to the predefined destination and releases the object. As the controller software is modular, any new command can be incorporated by modifying the firmware using Flash magic ISP programming. The pick and place application code is executed upon pressing the P\&P key on key matrix with simultaneous motion of all fingers for grasping and releasing an object placed within the volume of work space $(8 \mathrm{~cm}$ diam) of the study table. Home position key command code also runs with simultaneous motion of all fingers to open position for keeping the maximum work space.

\section{RESULTS}

The authors have constructed and tested a four fingered dextrous robot hand with a semianthropomorphic design as shown in Fig. 4 with functional block diagram. To achieve a high degree of modularity the hand consists of four identical fingers. The current arrangement shows three fingers and an opposing thumb. Each finger shows up one degree of freedom base joint with intersecting axes for curling motion and for abduction/adduction driven by one tendon actuated by single reversible geared DC motor. The finger joints are actuated by specially designed pulleys and idlers. The robot hand system can perform a finger tip grasp of a screwdriver and an enclosing grasp of a tin. The controlling software has been written in 8051 Assembly language to control it. 


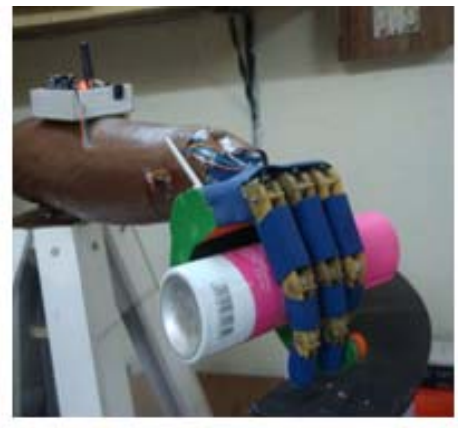

(a)

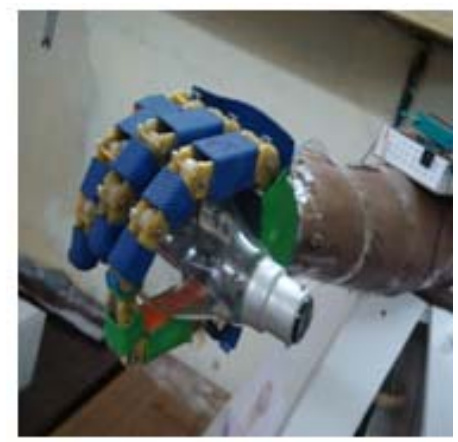

(b)

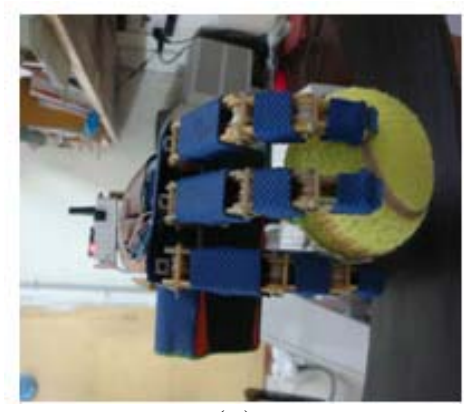

(c)

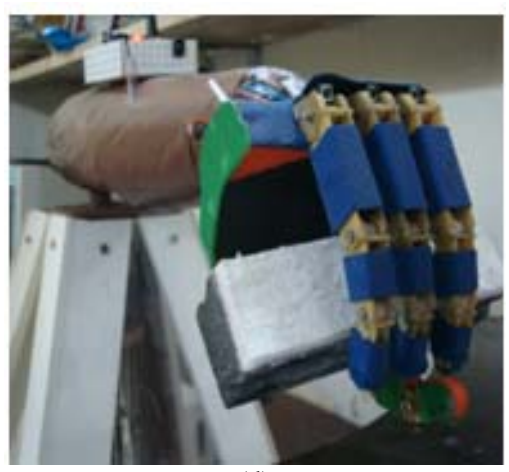

(d)

Fig. 7:FFRH grasping a variety of objects. (Tin, Bulb, Ball and Duster) (a) Cylindrical Tin (b) Electric bulb (c) Ball (d) Duster
The hand weighs approximately 4 Kgs. and the motors are currently mounted on the base of the robot.

To test the grasping ability of the hand, it was made to grasp different objects, each having different shape, size, surface conditions and hardness. The object was held so that the center of mass was within the workspace volume of the thumb and fingers and oriented to grasp so that the major axis of the object was parallel to the palm and aligned with the fingers. Once the objects were crudely positioned in the work space of hand, the hand was issued a pick and place command from the $5 \times 5$ key matrix of the FFRH. Different objects such as (a) Tin (b) Electric bulb (c) ball and (d) Duster are selected for object hunting and pick/place tasks. Figure 7 shows grasping of these objects.

The performance specifications of microcontrollerbased FFRH system that is implemented and tested are as follows: Maximum payload is $1 \mathrm{Kg}$., Object topology is arbitrary, independent degrees of freedom are 6, maximum diameter of work space sphere is 120 $\mathrm{mm}$ and minimum diameter of work space sphere is 30 $\mathrm{mm}$. An interesting aspect of this design is that the ranges of weight can be increased by adding more powerful motors and cables of higher tensile strength. Since these motors are mounted remotely, they do not add to the load of the manipulator. This enables the hand to be configured for the application by the selection of the appropriate motors. To test size restrictions on objects, experiments are performed at grasping spherical objects.

\section{DISCUSSION}

In the last few years, advanced robotic hands, with various fingers capable of different movements, have been developed, which have potential for use in the commercial, industrial, medical and prosthetic field. For example, the Stanford/JPL hand consists of 3 fingers, each with 3 Degrees Of Freedom(DOF), controlled by 12 actuators ; the Utah/MIT hand-one of the greatest achievements in the development of an anthropomorphic robotic hand-has 4 fingers, each with 4 DOF and 32 actuators; the Belgrade/USC hand consists of 5 fingers and 4 motors. However, the versatility and the functionality of an artificial hand is not determined only by the degree of mechanical complexity, that is, the number of DOF available; another very important issue is the presence of a sensorial system that can enable the grip to be optimized and tasks to be carried out efficiently and rapidly with either eye-in-hand or hand-eye coordination. Multi-fingered robot hand has been developed in our laboratory environment as an attempt 
to mimic human hand functionality with an eye-in-hand for simplified object hunting. The hand is controlled using a simple proportional control strategy from 8-bit micro-controller ports and relay drivers for all the joints. While each digit/finger of FFRH has one DOF, two more DOF are used for wrist control and base control. The achievement of this hand is to demonstrate reliable grasping with inexpensive mechanisms and push-button keys, reed switches and IR sensors for closed loop control. The authors have demonstrated that this hand can pick and place a variety of objects with different surface characteristics and shapes without having to reconstruct a surface description of the object.

\section{CONCLUSION}

The main features of designing and developing a dexterous robot hand as a modular system are outlined in this study. The application of robotics systems in unstructured servicing environments requires dextrose manipulation abilities and facilities to perform complex remote operations in a very flexible way. Therefore we have developed a multisensory articulated four fingered hand, where all actuators are integrated in the hand's base plate. After a brief description of the hand and it's wireless feedback sensorial logic, the hardware and software architecture are outlined with particular emphasis on flexibility and performance issues. The hand is typically controlled through a key matrix for all joints including pick and place as well as home position drive. The hand design is based on connected differential mechanisms and has been designed to be inexpensive, mechanically simple and easy to control. The tendoning system of the differential mechanism provides the hand with the ability to conform to object topology and therefore providing the advantage of using a simple control algorithm. The control algorithm used by FFRH is extremely simple as was the goal of the design. The controller takes a high-level command from the user key matrix and produces a successful grasp of the object that is within the workspace of the hand. Unlike most robot hands which use joint position measurement for control feedback, it uses only inexpensive simple contact/IR sensors. These sensors are being used primarily due to cost considerations, but have certain limitations. The advantage of designed robot hand is its simplicity and inexpensive. The FFRH system was designed as a first step in a future project that will integrate vision and grasping to control a robot to grasp objects and the future work will use a simple stereo vision system to determine the topology of the desired object and to visually move the robot hand so that the desired object is aligned within the workspace of the hand. As the robot hand system moves, the contact switches/IR sensors will be monitored to determine the successful grasping. This method is relatively simpler than the hugging algorithm presented for a robot hand configured as Graspar which relies on dense contact sensing (Tai, 2008; Parasuraman, 2008). The total robot hand system has independent control of finger joints of each finger leading to flexible control of finger motion as compared to the three fingered grasper (Goldfeder et al., 2009). Figure 7 shows the robot hand grasping different objects of different geometry.

In summary the key features of the FFRH are: (a) 360 degrees of Base rotation in both directions: wiring complexity is eliminated. workspace is increased, (b) two Relays instead of H-Bridge for motor direction control: simple and reliable, (c) sensors feedback by Radiometrix RF TX/RX modules avoiding wiring complexity, (d) only one motor for three joint controls of each finger in either direction, (e) simplified Robot eye by Infra red sensors for faster object detection without much Hunting time. (f) Ease of use and smaller Size and less Weight with good repeatability and payload of $1 \mathrm{Kg}$ of arbitrary Object shapes. The presentation is focused on efficient control architecture. It shows flexibility and computational power to meet even future requirements for autonomous manipulation. Future activities will focus on the implementation of powerful grasping strategies, further miniaturization and further improvement of mechanical and electrical reliability. The hand is supposed to become a central component for the development of future robonauts, but also aims at being used as prosthesis at least long-term.

\section{REFERENCES}

Cho, K.J., J. Rosmarm and H. Asada, 2006. Design of vast DOF artificial muscle actuators with a cellular array structure and its application to a five fingered robotic hand. Proceedings of the IEEE International Conference on Robotics and Automation, Orlando, Florida, May 15-19, Orlando, FL, pp: 2214-2219. DOI: 10.1109/ROBOT.2006.1642032

Ciocarlie, M.T. and P.K. Allen, 2009. Hand posture subspaces for dexterous robotic grasping. J. Robotics Res., 28: 851-867. DOI: 10.1177/0278364909105606

Goldfeder, C., C. Mater, J. Peretzman, H. Dang and P.K. Allen, 2009. Data-driven grasping with partial sensor data. Proceeding of the IEEE/RSJ International Conference on Intelligent Robots and Systems, Oct. 10-15, St. Louis, USA, pp: 12781283. DOI: 10.1109/IROS.2009.5354078 
Grecu, V., N. Dumitru and L. Grecu, 2009. Analysis of human arm joints and extension of the study to robot manipulator. Proceedings of the International MultiConference of Engineers and Computer Scientists, Mar. 18-20, Regal Kowloon Hotel, Tsimshatsui, Kowloon, Hong Kong, pp: 13481351.

http://www.iaeng.org/publication/IMECS2009/IM ECS2009_pp1348-1351.pdf.

Liu, H., K. Wu, P. Meusel, N. Seitz and G. Hirzinger et al., 2008. Multisensory five-finger dexterous hand: The DLR/HIT Hand II. Proceedings of the IEEE/RSJ International Conference Intelligent Robots and Systems, Sept. 22-26, Acropolis Convention Center, Nice, France, pp: 3692-3697. DOI: 10.1109/IROS.2008.4650624

Mayuko M. and S. Koichi et al., 2010. Development of power robot hand with shape adaptability using hydraulic mckibben muscles. Proceedings of IEEE International Conference on Robotics and Automation, May 3-7, Anchorage, Alaska, USA., pp: 1162-1168. 10.1109/ROBOT.2010.5509489

Odeh, S., R. Faqeh, L.A. Eid and N. Shamasneh, 2009. Vision-based obstacle avoidance of mobile robot using quantized spatial model. Am. J. Eng. Applied Sci., 2 : 511-619. 10.3844/ajeassp.2009.611.619
Parasuraman, S., 2008. Kinematics and control system design of manipulators for a humanoid robot. World Acad. Sci., Eng. Technol., 39: 7-13. http://www.akademik.unsri.ac.id/download/journal /files/waset/v39-2.pdf

Tai, E., 2008. Design of an anthropomorphic robotic hand for space operations. M.S. Thesis, Department of Aerospace Engineering, University of Maryland, USA., pp: 129. http://gradworks.umi.com/14/46/1446175.html

Wu, K., S. Fan, Y. Zhang, Z. Chen and H. Liu et al., 2010. Research of a novel miniature tactile sensor for five-finger dexterous robot hand. Proceedings of the IEEE International Conference on Mechatronics and Automation, Aug. 4-7, Xian, China, pp: 422- 427. DOI: 10.1109/ICMA.2010.5589147

Xu, K., R.E. Goldman, J. Ding, P.K. Allen, D.L. Fowler et al., 2009. System design of an insertable robotic effectors platform for Single Port Access (SPA) surgery. Proceeding of the IEEE International Conference Intelligent Robots and Systems, Oct. 10-15, St Louis, pp: 5546-5552. DOI: 10.1109/IROS.2009.5354028 